D) Check for updates

Cite this: Analyst, 2018, 143, 2002

Received 23rd January 2018, Accepted 11th April 2018

DOI: $10.1039 / c 8 a n 00131 f$

rsc.li/analyst

\section{Engineering molecularly imprinted polymers (MIPs) for the selective extraction and quantification of the novel psychoactive substance (NPS) methoxphenidine and its regioisomers $\uparrow$}

\author{
J. W. Lowdon, ${ }^{a}$ S. M. O. Alkirkit, ${ }^{a}$ R. E. Mewis, (D) ${ }^{a}$ D. Fulton, ${ }^{b}$ C. E. Banks, (D) \\ O. B. Sutcliffe (iD $* t^{a, d}$ and M. Peeters (iD $* t^{a}$
}

In this communication, we present the first developed Molecularly Imprinted Polymers (MIPs) for the specific detection of a New Psychoactive Substance (NPS); namely, methoxphenidine (MXP) and its regioisomers. Selectivity of the MIP towards MXP is studied by analysing mixtures and an acquired street sample with High Performance Liquid Chromatography coupled to UV detection. The study demonstrates that the engineered polymers selectively extract MXP from heterogeneous samples, which makes for a very powerful diagnostic tool that can detect traces of MXP in complicated NPS samples.

\section{Introduction}

Methoxphenidine (MXP, Scheme 1) is a dissociative anaesthetic of the diarylethylamine class that acts as an agonist on the $N$-methyl-D-aspartate receptors in the central nervous system $^{1}$ and has been implicated in a number of fatalities. ${ }^{2}$ While the Psychoactive Substances Act (2016) controls the distribution and production of diphenidine and its derivatives (such as MXP) in the United Kingdom, the global prevalence of Novel Psychoactive Substances (NPS) is on the rise year on year. ${ }^{3}$ Recently, there have been increasing levels of concern about the onset of acute and chronic psychopathological manifestations that are associated with the intake of NPS that have

${ }^{a}$ Manchester Metropolitan University, Faculty of Science and Engineering, School of Science and the Environment, Division of Chemistry and Environmental Science, Chester Street, Manchester M1 5GD, UK. E-mail: o.sutcliffe@mmu.ac.uk, m.peeters@mmu.ac.uk; Tel: +44(0)1612471450

${ }^{b}$ Newcastle University, School of Chemistry, Bedson Building, Newcastle Upon Tyne, NE1 $7 R U, U K$

${ }^{c}$ Manchester Metropolitan University, Faculty of Science and Engineering, School of Healthcare Science, Chester Street, Manchester M1 5GD, UK

${ }^{d}$ MANchester DRug Analysis and Knowledge Exchange (MANDRAKE), Manchester Metropolitan University, Faculty of Science and Engineering, Chester Street, Manchester M1 5GD, UK

$\dagger$ Electronic supplementary information (ESI) available. See DOI: 10.1039/ c2dt12242a

$\$$ These authors share senior and corresponding authorship.
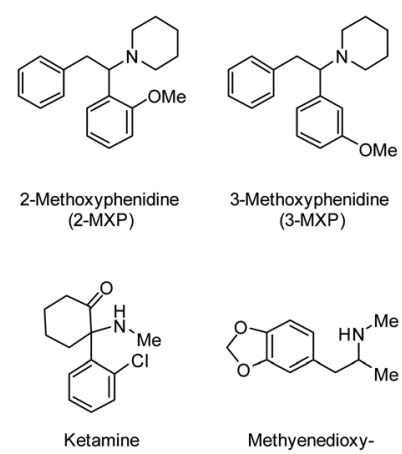

3-Methoxyphenidine (3-MXP)
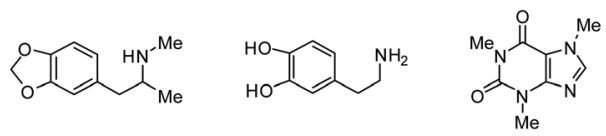

Methyenedioxymethamphetamine (MDMA)
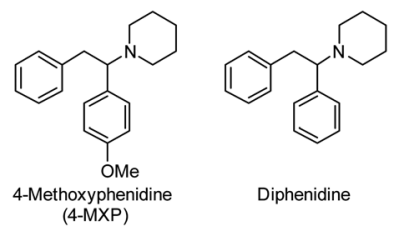

Diphenidine

Dopamine

Scheme 1 MIPs were developed for the MXP isomers and testing against a variety of other compounds to test specificity and selectivity.

MXP as its main dissociative ingredient. ${ }^{3}$ The difficulty herein is that NPS are sold under a variety of brand names with unknown composition, and therefore it might be that encountered side effects are caused by either regioisomeric impurities or common adulterants and substances used to cut NPS with. ${ }^{4}$ Thus, there is a significant analytical challenge ahead to selectively extract 2-MXP from samples and determine the forensic fingerprint of NPS samples. Chromatographic techniques show great promise in that respect but matrix components can interfere with accurate quantification and invalidate the whole analysis. ${ }^{5,6}$ Sample preparation and pre-treatment is considered the bottleneck of the analytical process, which can be overcome by the use of Molecularly Imprinted Polymers (MIPs). MIPs are polymeric synthetic receptors with a high affinity for their template and this makes them extremely suitable for the selective extraction of targets from complex matrices. ${ }^{7,8}$ Their advantages include low-cost, stability under extremes of temperature and $\mathrm{pH}$, re-usability, and straightforward preparation process. ${ }^{9,10}$ Several companies offer commercial cartridges packed with MIPs for determination of targets in a variety of samples, but their use for the extraction and purification of NPS remains unexplored. This niche in the 
market is most likely due to limited access to pure compounds and time that is required to prepare and optimize MIPs for novel targets. ${ }^{11}$ For the first time, we will report on the synthesis of a MIP for MXP isomers. First, all MXP isomers were synthesized according to a Barbier-type reaction. ${ }^{12}$ Subsequently, the MIP was templated with 2-MXP as this is the most prevalent isomer in seized bulk forensic samples that are marketed as "methoxphenidine". The binding affinity of the MIP towards all positional MXP isomers and similar compounds in solutions is evaluated by optical batch rebinding experiments. All these compounds are listed in Scheme 1.

A novel High Performance Liquid Chromatography (HPLC) method was developed for the separation of the different MXP isomers from complicated samples. HPLC employing UV detection was then used to evaluate the NPS mixtures and an acquired street sample. This demonstrates that the developed MIP selectively captures MXP in the presence of an excess of similar NPS and other common adulterants.

MIPs are versatile and can be tailored towards other targets; which makes the engineered polymers a powerful tool for the quantification and extraction of traces of NPS in heterogeneous samples. It would allow to forensically fingerprint seized NPS samples, which will provide useful data on the toxicology associated with these substances.

\section{Experimental}

\subsection{Reagents}

Ethylene glycol dimethacrylate (EGDMA), divinylbenzene (DVB), trimethylolpropane trimethacrylate (TRIM) and methacrylic acid (MAA) were purchased from Acros (Loughborough, UK). Prior to polymerization, the stabilizers in the monomers and crosslinker monomers were removed by passing the solutions over a column packed with alumina. Initiator 4,4'-azobis(4-cyanovaleric acid), ketamine hydrochloride, 3,4-methylenedioxymethamphetamine hydrochloride (MDMA), caffeine and solvents were obtained from Sigma Aldrich (Gillingham, UK). Diphenidine and the positional isomers of methoxphenidine (Scheme 1) were prepared, under UK Home Office license, as their corresponding hydrochloride salts using the procedure reported by Geyer et al. ${ }^{5}$ The street sample was obtained from BRC Fine Chemicals (http://www.brc-finechemicals.com) prior to the legislative change. The Psychoactive Substances Act (2016), which came in effect on the $26^{\text {th }}$ May 2016, makes it an offence to manufacture, supply, possess and import and export psychoactive substances named in this Act, which includes MXP derivatives and their isomers. Structure and purity (>99\%) of the target compounds was determined by NMR analysis and GCMS respectively. ${ }^{5}$ All solutions were prepared with deionized water of a resistivity of $18.2 \Omega \mathrm{cm}$ or with home-made phosphate buffered saline $(1 \times \mathrm{PBS})$ solutions.

\subsection{MIP synthesis}

MIPs were developed using styrene and methacrylic acid as monomers. Styrene, which interacts with the benzene rings of
Table 1 Composition of all MIP mixtures

\begin{tabular}{llllll}
\hline & MIP 1 & MIP 2 & MIP 3 & MIP 4 & MIP 5 \\
\hline 2-MXP (mmol) & 0.17 & 0.17 & 0.17 & 0.17 & 0.17 \\
Styrene (mmol) & - & - & 1.82 & 1.82 & - \\
MAA (mmol) & 1.82 & 1.82 & - & - & 1.82 \\
TRIM (mmol) & 3.64 & - & - & - & - \\
EGDM (mmol) & - & 3.64 & - & 3.64 & - \\
DVB (mmol) & - & - & 3.64 & - & 3.64 \\
\hline
\end{tabular}

MXP, resulted in the formation of polymers that were partially soluble in water and therefore not suitable for detection purposes. Subsequently, methacrylic acid was explored as a monomer with various crosslinkers including EGDM, TRIM and DVB. The composition is described in Table 1. The initiator azocyanovaleric acid was kept at $50 \mathrm{mg}$. In all mixtures, $3 \mathrm{~mL}$ of DMSO as the porogen was used. 2-MXP was used as $\mathrm{s}$ a template since this is the most prevalent isomer in street samples and has been linked to toxicity, meaning identification and detection of this isomer is particularly relevant. The polymer with the optimal composition was repeated with 4-MXP as template but this did not have a significant effect on the rebinding results. ${ }^{4}$ Monomer to template ratios of approximately 10:1 were used since lower values did not result in significant binding.

The MIP with the highest binding affinity was prepared as follows. First, a mixture of the functional monomer MAA $(1.82 \mathrm{mmol})$ and template 2-MXP $(0.17 \mathrm{mmol})$ were dissolved in $3 \mathrm{~mL}$ of DMSO. Subsequently, the crosslinker molecule EGDM (3.64 mmol), initiator 4,4-azobis(4-cyanovaleric acid) (50 mg) were added. This mixture was sonicated for five minutes and subsequently degassed with $\mathrm{N}_{2}$ before commencing the polymerization. The mixture was heated to $65{ }^{\circ} \mathrm{C}$ for $12 \mathrm{~h}$, which allowed full completion of the polymerization. The final polymer was ground and sieved to obtain microparticles with sizes smaller than $100 \mu \mathrm{m}$, as was confirmed by Scanning Electron Microscopy analysis (ESI $1 \dagger$ ). The template 2-MXP was then removed from the powders by continuous Soxhlet extraction with 50/50 mixtures of acetic acid and methanol and a 50/ 50 mixtures of methanol and water. Extraction was performed until no traces of 2-MXP were found in the UV-vis spectrum (wavelength $=250 \mathrm{~nm}$ ), confirming complete removal of the template. Non-Imprinted Polymers (NIPs) were synthesized accordingly but without the presence of the template molecule. Finally, powders were dried overnight in an oven at $100{ }^{\circ} \mathrm{C}$. Stability of the MIPs at elevated temperatures was determined by ThermoGravimetric Analysis, which showed that the polymers were stable until $220{ }^{\circ} \mathrm{C}$ (ESI $2 \dagger$ ).

\subsection{Batch rebinding experiments}

Optical batch rebinding experiments were evaluated with an Agilent 8453 spectrophotometer (Stockport, United Kingdom). For each experiment, $20 \mathrm{mg}$ of MIP or Non-Imprinted Polymer (NIP, used as reference) powder was added to $5 \mathrm{~mL}$ of aqueous solution with 2-MXP concentrations between 0-0.7 mM. The $\mathrm{pH}$ of the solutions was adjusted in a range from 3-10 by 
addition of a $\mathrm{HCl}$ solution or $\mathrm{NaOH}$ solution $(0.1 \mathrm{mM})$. The measurement at $\mathrm{pH} 7$, which gave the highest binding of MXP to the MIP, was also performed in a PBS solution to evaluate the influence of matrix compounds (salts) on binding. The resulting suspensions were placed on an orbital shaker (125 $\mathrm{rpm}$ ) for $90 \mathrm{~min}$ at room temperature. With a time dependent experiment, it was determined that this was the optimum rebinding time for all isomers (ESI $4 \dagger$ ). After filtration, the free concentration of 2-MXP in the filtrate was determined by UVvis spectroscopy and HPLC analysis and binding isotherms were constructed. As a measure of specificity and in order to compare the different MIP compositions, the imprint factor (IF) was determined at a free concentration of the template $\left(C_{\mathrm{f}}\right)=0.05 \mathrm{mM}$. To determine whether the MIP could selectively extract MXP isomers from mixtures, selectivity tests were performed with diphenidine, a NPS with a similar structure to the template, illicit substances with similar biological function including ketamine and 3,4-methylenedioxymethamphetamine (MDMA), the neurotransmitter dopamine, and finally, caffeine, a common adulterant in street samples.

\subsection{Extraction of MXP isomers from complex samples evaluated by HPLC analysis}

Binding isotherms of the MIP to solutions containing pure compounds were determined by both UV-vis and HPLC in aqueous solutions $(\mathrm{pH}=7)$. High-performance liquid chromatography was performed with an integrated Agilent HP Series 1100 Liquid Chromatograph (Agilent Technologies, Wokingham, UK) fitted with an in-line degasser, 100-place auto-injector and diode array UV absorbance detector (DAD, $220 \mathrm{~nm}$ ). Data analysis (HPLC) was carried out using Chemstation for LC (Ver. 10.02) software (Agilent Technologies, Wokingham, UK). The flow rate was $1.0 \mathrm{~mL}$ $\min ^{-1}$ with an injection volume of $10 \mu \mathrm{L}$. Three replicate injections of each solution (standards and samples) were performed. The stationary phase (ACE 5 C18-AR, $150 \mathrm{~mm} \times$ $4.6 \mathrm{~mm}$ i.d., particle size: $5 \mu \mathrm{m}$ ) used in the study was obtained from HiChrom Limited (Reading, UK). The column was fitted with a guard cartridge (ACE 5 C18-AR) and maintained at an isothermal temperature of $50{ }^{\circ} \mathrm{C}$ with an Agilent HP Series 1100 column oven with a programmable controller (Agilent Technologies, Wokingham, UK). The mobile phase employed in this study was acetonitrile: $20 \mathrm{mM}$ ammonium acetate (55: 45\% v/v). Simulated test samples containing different mixtures/ratios of diphenidine, the MXP isomers, caffeine, MDMA and ketamine were studied. The applicability of the MIP developed herein was evaluated using both these simulated test samples and a street sample obtained from an on-line vendor.

\section{Results}

\subsection{Batch rebinding results}

It was not possible to determine isotherms for MIPs 3-4 with styrene as monomer. The synthesized polymers were extremely hydrophobic which was not compatible with detection in aqueous solutions. Binding isotherms were constructed for MIPs using MAA as monomer and with varying crosslinker monomers (MIP 1,2,5) composition. To determine the specificity of the various MIPs in a quantitative manner, the IF $\left(S_{\mathrm{b}}\right.$ $\mathrm{MIP} / S_{\mathrm{b}}$ NIP at a certain concentration) was calculated at $C_{\mathrm{f}}=$ $0.05 \mathrm{mM}$ for aqueous 2-MXP solutions with $\mathrm{pH}=7$. The data was fitted $\left(R^{2}=0.95\right)$ with an allometric fit $\left(y=A x^{b}\right)$ that is typical for heterogeneous MIP distributions. ${ }^{13}$ MIP 2 with EGDM as crosslinker monomer had an IF of 2.0, while MIP 1 with TRIM (IF = 1.3) and MIP 5 with DVB (IF = 1.2) as crosslinker monomers achieved less specificity. This could be due to the good compatibility of EGDM with the monomer and the limited flexibility of the crosslinker molecule, which is known to enhance binding. Therefore, in further experiments we continued with the MIP with EGDM as crosslinker monomer since these polymers exhibited the highest affinity towards 2-MXP. Subsequently, the influence of $\mathrm{pH}$ on binding of 2-MXP to the optimized MIP particles was evaluated (Fig. 1). Error bars were determined by taking the standard deviation over three individual experiments. It is observed that binding to the MIP is significantly lower in acidic environments. This is because at $\mathrm{pH}$ values below the $\mathrm{p} K_{\mathrm{a}}$ (6.5) of methacrylic acid, the acid is predominantly in its neutral form and hydrogen bonding to the nitrogen functionality or methoxy group of MXP is unlikely to occur. Neutral environments enhance binding as both the monomer and template are in its ionized form. The binding capacity at $\mathrm{pH}=8$ is slightly slower than for $\mathrm{pH} 7$, but overall the same trend line is followed. At $\mathrm{pH}$ 10, the affinity is overall lower, which could potentially due to less ionic interactions between monomer and template.

To determine IF values, data are fitted with an allometric fit $(\mathrm{pH} 3,4,8$ 10) or a linear curve ( $\mathrm{pH} 7)$. A linear fit was used

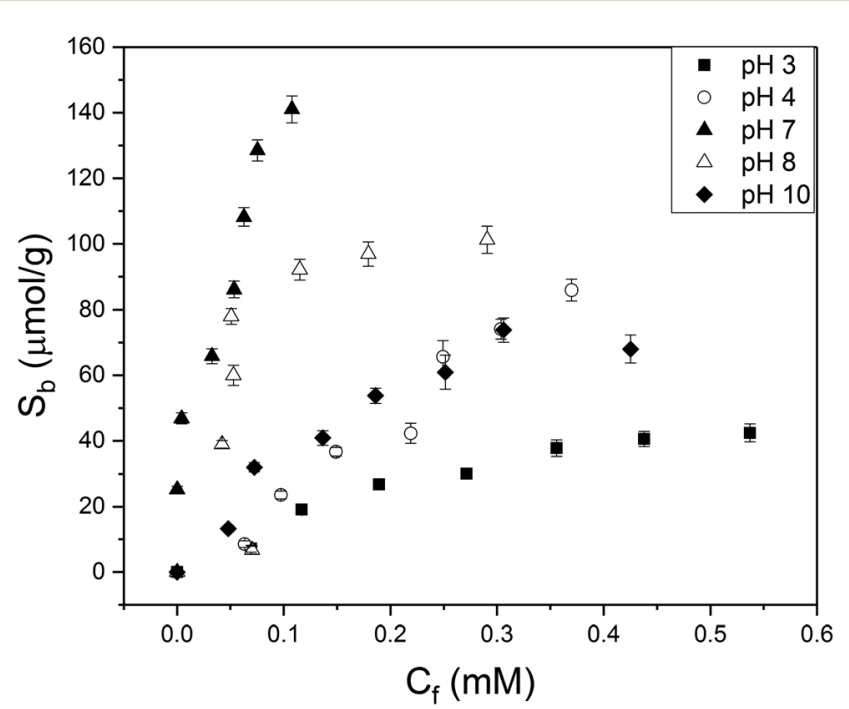

Fig. 1 Binding isotherms of MIPs upon exposure to aqueous 2-MXP solutions at $\mathrm{pH} 3$ (solid squares), $\mathrm{pH} 4$ (open circles), $\mathrm{pH} 7$ (solid triangles), $\mathrm{pH} 8$ (open triangles) and $\mathrm{pH} 10$ (solid diamonds). Error bars correspond to the standard deviation taken over three individual batch rebinding experiments. 
for $\mathrm{pH}$ because no saturation of binding sites was observed in this particular concentration range. $R^{2}$ values of minimal 0.94 were achieved, demonstrating a good match between the observed and predicted data. The data for binding to the NIP and the fit parameters that were used in order to determine IF values, are provided in the ESI (S5, S6 and S7 $\dagger$ ).

The maximum number of binding sites $\left(N_{\text {tot }}\right)$ in aqueous solutions of $\mathrm{pH}=7$ was determined to be $190 \pm 10 \mu \mathrm{mol} \mathrm{g}^{-1}$ according to the Freundlich isotherm. In PBS solution, IF was not affected but number of binding sites was reduced $\left(N_{\text {tot }}=\right.$ $130 \pm 15 \mu \mathrm{mol} \mathrm{\textrm {g } ^ { - 1 }}$ ) due to the presence of other ions. Subsequently, the selectivity of the MIP towards the positional isomers of MXP and other drug compounds were studied in aqueous solutions (Fig. 2). The selectivity measurements were performed in duplicate with an average deviation of $5 \%$ in the results.

It was determined that the MIP templated for 2-MXP was capable of extracting all MXP isomers with similar binding capacities at $C_{\mathrm{f}}=0.05 \mathrm{mM}\left(\sim 190 \pm 10 \mu \mathrm{mol} \mathrm{g}^{-1}\right)$. The MIP had a much lower affinity for diphenidine, which of all NPS is the most similar to MXP as it has the same carbon skeleton but lacks the OMe group. This illustrates that not just the nitrogen functionality, but also the methoxy group has a crucial role in

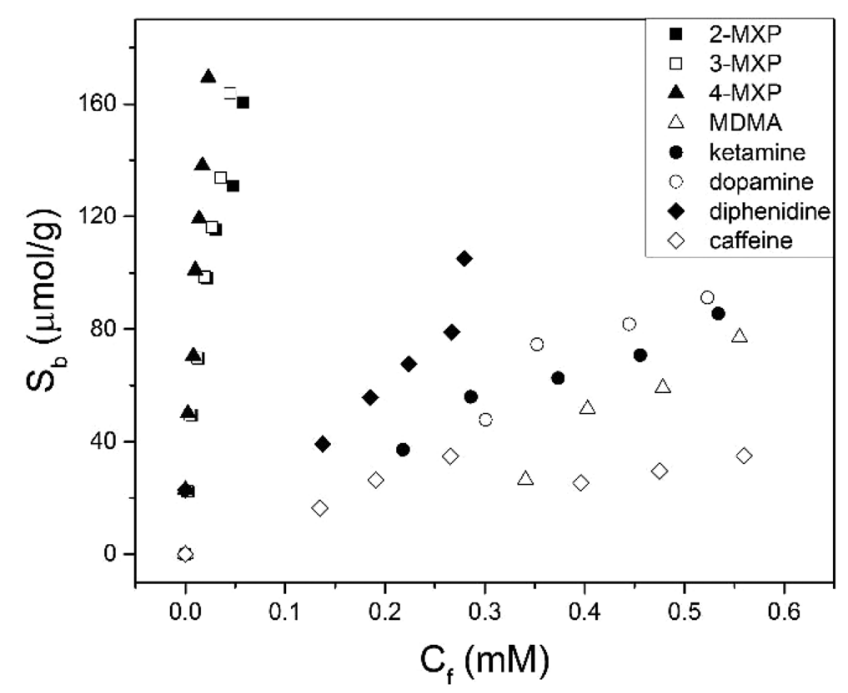

Fig. 2 Binding isotherms of MIPs templated for 2-MXP upon exposure to aqueous solutions ( $\mathrm{pH}=7$ ) of 2-MXP (solid squares), 3-MXP (open squares), 4-MXP (solid triangles), ketamine (solid circles), dopamine (open circles), diphenidine (solid diamonds) and caffeine (open diamonds). achieving high binding affinity for the MXP isomers. The position of the methoxy group is not of significant relevance, otherwise differences in binding between the various MXP isomers would be expected. At $C_{\mathrm{f}}=0.05 \mathrm{mM}$, the MIP particles absorbed $120 \pm 10 \mu \mathrm{mol} \mathrm{\textrm {g } ^ { - 1 }}$ of all MXP isomers but only $30 \mu \mathrm{mol} \mathrm{g}{ }^{-1}$ of diphenidine. Binding to the MIP particles of other illicit substances such as ketamine $\left(25 \pm 2 \mu \mathrm{mol} \mathrm{g}^{-1}\right)$ or MDMA (19 $\left.\pm 3 \mu \mathrm{mol} \mathrm{g}^{-1}\right)$, and caffeine, a common adulterant $\left(15 \pm 1 \mu \mathrm{mol} \mathrm{g}{ }^{-1}\right)$, were significantly lower under identical conditions. It is not possible to discriminate binding of the different MXP isomers and other illicit substances by means of UV-vis spectroscopy. Therefore, HPLC coupled to UV-vis spectroscopy was used in further experiments, which was used to gain valuable insight in to the binding of different chemical compounds to the MIP in mixtures.

\subsection{Batch rebinding results evaluated by HPLC coupled to UV}

It was determined that the binding capacity of the MIP towards MXP isomers was $\sim 170 \mu \mathrm{mol} \mathrm{g}^{-1}$ for the experiments performed by HPLC, which is similar to what was obtained with UV-vis spectroscopy in the same range of $C_{\mathrm{f}}(190 \pm$ $10 \mu \mathrm{mol} \mathrm{g}{ }^{-1}$ ). In Table 2 , the binding capacity and percentage recovery for pure samples and mixtures of MXP isomers is recorded.

It can be observed that the MIP has no significant preference towards a particular isomer and in pure solutions, a high percentage recovery ( $>90 \%)$ was achieved. In mixtures, binding is significantly lowered which can be explained by the fact that binding to the MIP is an equilibrium process and competitive inhibition by similar substrates (such as isomers) can occur. This could be overcome by either increasing the binding time or the amount of MIP particles.

The full isotherms for the MIPs towards exposure of 2-, 3and 4-MXP are described in ESI S-6 and S-8, $\dagger$ which also include the response of a NIP to 2-MXP.

To determine the extraction capability of the MIP towards the MXP isomers in heterogeneous samples, mixtures with varying ratios of 2-MXP and other NPS or illicit substances were prepared (Table 3 ).

These mixtures contained compounds which 2-MXP is commonly known to be "cut" with such as diphenidine, ketamine, MDMA and the common adulterant caffeine. Percentage extraction of each substrate was calculated by comparing substrate absorbance before and after the MIP was introduced for 90 minutes (Table 4). Error bars were recorded by taking the standard deviation over three individual HPLC measurements.

Table 2 Binding capacity $\left(\mu \mathrm{mol} \mathrm{g}{ }^{-1}\right.$ ) and percentage recovery (\%) of MXP in pure aqueous solutions and in aqueous mixtures with equimolar ratios of MXP isomers. Error bars were determined by using the standard deviation over three individual experiments

\begin{tabular}{lllll}
\hline Target & $\begin{array}{l}\text { Binding capacity } \\
\text { solution }\left(\mu \mathrm{mol} \mathrm{g}^{-1}\right)\end{array}$ & $\begin{array}{l}\text { Binding capacity } \\
\text { mixture }\left(\mu \mathrm{mol} \mathrm{g}^{-1}\right)\end{array}$ & $\begin{array}{l}\text { Percentage recovery } \\
\text { solution }(\%)\end{array}$ & $\begin{array}{l}\text { Percentage recovery } \\
\text { mixture }(\%)\end{array}$ \\
\hline 2-MXP & $168 \pm 5$ & $114 \pm 7$ & $91 \pm 1$ & $64 \pm 2$ \\
3-MXP & $169 \pm 10$ & $127 \pm 5$ & $94 \pm 2$ & $74 \pm 1$ \\
4-MXP & $171 \pm 8$ & $129 \pm 8$ & $97 \pm 2$ & $75 \pm 2$
\end{tabular}


Table 3 Ratios of substrates in simulated test samples

\begin{tabular}{|c|c|c|c|c|c|c|}
\hline Substrate & Ratio $1\left(\mathrm{mg} \mathrm{mL}^{-1}\right)$ & Ratio $2\left(\mathrm{mg} \mathrm{mL}^{-1}\right)$ & Ratio $3\left(\mathrm{mg} \mathrm{mL}{ }^{-1}\right)$ & Ratio $4\left(\mathrm{mg} \mathrm{mL}{ }^{-1}\right)$ & Ratio $5\left(\mathrm{mg} \mathrm{mL} \mathrm{m}^{-1}\right)$ & Ratio $6\left(\mathrm{mg} \mathrm{mL}{ }^{-1}\right)$ \\
\hline Caffeine & 1 & 0.5 & 1.5 & 1.5 & 1.5 & 1 \\
\hline MDMA & - & - & 0.5 & - & 0.5 & - \\
\hline Ketamine & - & - & - & 0.5 & 0.5 & - \\
\hline 3-MXP & - & - & - & - & - & 0.5 \\
\hline
\end{tabular}

Table 4 Percentage extraction of varying compositions of simulated NPS samples. Error bars correspond to the standard deviation taken over three individual HPLC experiments

\begin{tabular}{|c|c|c|c|c|c|c|}
\hline \multirow[b]{2}{*}{ Substrate } & \multicolumn{6}{|c|}{ Percentage extraction (\%) } \\
\hline & Ratio 1 & Ratio 2 & Ratio 3 & Ratio 4 & Ratio 5 & Ratio 6 \\
\hline 2-MXP : MIP & $76 \pm 2$ & $79 \pm 4$ & $82 \pm 5$ & $82 \pm 3$ & $79 \pm 2$ & \multirow{2}{*}{$\underset{a}{77} \pm 4$} \\
\hline 2 MXP : NIP & $22 \pm 2$ & $22 \pm 3$ & $16 \pm 1$ & $15 \pm 1$ & $17 \pm 2$ & \\
\hline Caffeine & $11.4 \pm 2$ & $7.5 \pm 0.3$ & $18 \pm 4$ & $24 \pm 4$ & $23 \pm 4$ & $9 \pm 4$ \\
\hline Diphenidine & $28.0 \pm 0.6$ & $13 \pm 4$ & - & - & - & - \\
\hline MDMA & - & - & $26 \pm 1$ & - & $23 \pm 4$ & - \\
\hline Ketamine & - & - & - & $28 \pm 1$ & $19 \pm 3$ & - \\
\hline 3-MXP & - & - & - & - & - & $84 \pm 2$ \\
\hline
\end{tabular}

These results indicate that the presence of other adulterants or illicit substances had only limited effect on the extraction capability of the MIP towards 2-MXP since recoveries of $>75 \%$ were recovered in mixtures. Competitive binding of the different MXP isomers had a more significant impact on the binding capacity (see Table 2 where recoveries of $\sim 70 \%$ were recorded). The binding of 2-MXP to the NIP particles is limited (max $\sim 20 \%$ ), which is additional proof of the high selectivity of the engineered polymer particles. Furthermore, it has to be considered that since this is an in vitro test we have full control over the $\mathrm{pH}$ and ionic strength of the sample and simple sample pre-treatment can remove the effects of other common adulterants that are used to cut samples with (including sodium bicarbonate).

Finally, the composition of a street sample of $1.0 \mathrm{~g}$ MXP (acquired from Buy Research Chemicals UK prior to the legislative change, see ref. 6) was measured. After binding to the MIP for $90 \mathrm{~min}$, the HPLC analysis method determined that the sample was composed of $97 \pm 1 \% 2-\mathrm{MXP}, 3 \pm 1 \%$ of 3 -MXP and free of adulterants. This is in-line with previously conducted GC-MS analysis, which showed that the sample did not contain any adulterants and had high levels ( 99\%) of the 2-MXP isomer.

\section{Conclusions}

This is the first report of a MIP for a NPS, which were synthesized with 2-MXP as a template, styrene and MAA as the functional monomers and varying crosslinker monomers. It was determined using optical batch rebinding experiments that the binding of MXP in aqueous solutions to the MIP was
pH dependent. Neutral solutions, when both monomer and template are under ionized conditions, proved to be the optimal conditions and maximum binding capacities of all MXP isomers in the range of $170-190 \mu \mathrm{mol} \mathrm{g}{ }^{-1}$ were recorded by optical batch rebinding and chromatographic methods. Specificity of the MIP was retained in buffered solutions and IF values of $\sim 2.1$ were recorded, showing the affinity towards MXP. HPLC coupled to UV-vis spectroscopy was used to validate optical batch rebinding experiments and to study NPS mixtures. The developed HPLC procedure allows for a clear separation between the three MXP regioisomers, which is a novel method that has not been reported in the literature before. It was determined that there is high recovery of all MXP isomers in pure solutions ( $>90 \%)$. In mixtures with equimolar ratios of MXP isomers, competitive binding occurred and there was no preference over one specific isomer. Selective extraction of the MXP isomers was possible in mixtures with various ratios of the MXP isomers, and even in the presence of an excess of adulterants and other illicit substances. This shows that molecular imprinting is a powerful technology for the extraction and quantification of (traces) of psychoactive components in complicated samples. It is a promising tool for the use in forensic analysis, but also has the potential to be extended to environmental analytical analysis such as contamination of food and drinking water with psychoactive substances.

\section{Conflicts of interest}

The authors declare no conflicts of interest. 


\section{Acknowledgements}

We would like to thank François Nguyen and Taejun Lim for their assistance with the optical batch rebinding experiments.

\section{References}

1 J. Wallach, H. Kang, T. Colestock, H. Morris, Z. A. Bortolotto, G. L. Collingridge, D. Lodge, A. L. Halberstadt, S. D. Brandt and A. Adejare, PLoS One, 2016, 11, e0157021.

2 A. Helander, O. Beck and M. Baeckberg, Clin. Toxicol., 2015, 53, 446.

3 S. Beharry and S. Gibbons, Forensic Sci. Int., 2016, 267, 25.

4 S. Davies, T. Lee, J. Ramsey, P. I. Dargan and D. M. Wood, Eur. J. Clin. Pharmacol., 2012, 68, 435.

5 P. M. Geyer, M. C. Hulme, J. P. Irving, P. D. Thompson, R. N. Ashton, R. J. Lee, L. Johnson, J. Marron, C. E. Banks and O. B. Sutcliffe, Anal. Bioanal. Chem., 2016, 408, 8467.
6 F. G. Tamayo, E. Turiel and A. Martín-Esteban, J. Chromatogr., A, 2007, 1152, 32.

7 I. Chianella, A. Guerreiro, E. Moczko, J. S. Caygill, E. V. Piletska, I. M. P. De Vargas Sansalvador, M. J. Whitcombe and S. A. Piletsky, Anal. Chem., 2013, 85, 8462 .

8 B. Sellergren and C. J. Allender, Adv. Drug Delivery Rev., 2005, 57, 1733.

9 A. J. Hall, P. Manesiotis, M. Emgenbroich, M. Quaglia, E. De Lorenzi and B. Sellegren, J. Org. Chem., 2005, 70, 1732.

10 K. Haupt and K. Mosbach, Chem. Rev., 2000, 100, 2495.

11 A. Zurutuza, S. Bayoudhy, P. A. G. Cormack, J. Dambies, J. Deere, R. Bischoff and D. C. Sherrington, Anal. Chim. Acta, 2005, 542, 14.

12 E. Le Gall, C. Haurena, S. Sengmany, T. Martens and M. Troupel, J. Org. Chem., 2009, 74, 7970.

13 R. J. Umpleby, S. J. Baxter, A. M. Rampey, G. T. Rushton, Y. Z. Chen and K. D. Shimizu, J. Chromatogr., B: Anal. Technol. Biomed. Life Sci., 2004, 804, 141. 